\title{
Optimized immunohistochemical panel to differentiate myeloid sarcoma from blastic plasmacytoid dendritic cell neoplasm
}

\author{
Nikhil A Sangle ${ }^{1}$, Robert L Schmidt ${ }^{1}$, Jay L Patel ${ }^{2}$, L Jeffrey Medeiros ${ }^{3}$, \\ Archana M Agarwal ${ }^{1}$, Sherrie L Perkins ${ }^{1}$ and Mohamed E Salama ${ }^{1}$ \\ ${ }^{1}$ Department of Pathology, University of Utah Health Sciences Center, Salt Lake City, UT, USA; \\ ${ }^{2}$ Department of Pathology, University of Calgary, Alberta, Canada and ${ }^{3}$ Department of Pathology, \\ The University of Texas MD Anderson Cancer Center, Houston, TX, USA
}

\begin{abstract}
Myeloid sarcoma (MS) and blastic plasmacytoid dendritic cell neoplasm (BPDCN) can be difficult to distinguish morphologically, even with the use of extensive immunohistochemical studies. Three new research markers, myxovirus A (MXA), CLA/CD162, and CD303/BDCA-2, have been reported to be positive in BPDCN, but their clinical utility has never been tested. We compared these markers to other antibodies that have been used traditionally to distinguish MS from BPDCN to assess the utility of these newer antibodies in differential diagnosis. Formalin-fixed, paraffin-embedded tissue sections of 23 MS and 17 BPDCN cases were assessed using immunohistochemical analysis for CD4, CD14, CD33, CD43, CD56, CD68, CD123, CD163, myeloperoxidase, lysozyme, terminal deoxynucleotidyl transferase (TdT), T-cell leukemia 1 (TCL-1), MxA, cutaneous lymphocyte-associated antigen (CLA)/CD162, and blood dendritic cell antigen 2 (BDCA2)/CD303. We identified antibodies with a high predictive value of $\geq 90 \%$ and used these markers to develop an approach to classification using specific staining criteria. Diagnostic classification criteria were based on staining patterns of one or more of the seven markers. BPDCN was associated with positive staining for CD56, TdT, or TCL1, or negative staining for lysozyme. MS was associated with positive staining for lysozyme or myeloperoxidase, or negative staining for CD56, CD123, myxovirus, or TCL1. The immunohistochemical staining patterns observed using a panel that includes MPO, CD56, CD123, TCL1, TdT, and MXA, are predictive of MS or BPDCN. In this study, neither CD162 nor CD303 had good predictive value in distinguishing MS from BPDCN.
\end{abstract}

Modern Pathology (2014) 27, 1137-1143; doi:10.1038/modpathol.2013.238; published online 3 January 2014

Keywords: Myeloid sarcoma BPDCN immunohistochemical panel

Blastic plasmacytoid dendritic cell neoplasm (BPDCN), previously known as blastic NK-cell lymphoma or agranular CD4 + /CD56 + hematodermic neoplasm, was first described by Adachi et al ${ }^{1}$ and originate from precursors of plasmacytoid dendritic cells. ${ }^{2-5}$ BPDCN is often seen in elderly individuals, but a few cases in children are also reported in the literature. ${ }^{6}$ Lesions may be initially limited to the skin, with progression to a more generalized disease in the form of bone marrow

Correspondence: Associate Professor/Dr ME Salama, MD, Department of Pathology, Division of Hematopathology, University of Utah Health Sciences Center, Salt Lake City, UT 84132, USA.

E-mail: mohamed.salama@aruplab.com

Received 29 May 2013; revised 12 November 2013; accepted 14 November 2013; published online 3 January 2014 involvement and leukemic transformation in most cases. ${ }^{6}$ The skin manifestations are heterogeneous and include erythema, patches, papules, nodules, and ulcers. It is proposed that the skin tropism of BPDCN is likely related to the skin-homing properties of cutaneous lymphocyte-associated antigen (CLA/CD162). ${ }^{7}$

Myeloid sarcoma (MS), also referred to as granulocytic sarcoma or extramedullary myeloid tumor, shows a predilection for skin but also involves other extramedullary sites such as lymph nodes, paranasal sinuses, gingiva, and soft tissue. ${ }^{8}$ MS may initially manifest as skin lesions and can be associated with concurrent or antecedent acute leukemia in the bone marrow. MS shows a varying clinical spectrum in the skin, manifesting as an erythematous rash, urticaria, purpura, or maculopapular eruptions. This overlapping clinical spectrum of skin-based blastic 
neoplasms makes biopsy essential for diagnosis as each diagnosis carries specific clinical and prognostic implications. ${ }^{9-11}$

Histologically, both MS and BPDCN exhibit large atypical cells with high nuclear-to-cytoplasmic ratio, irregular nuclear membranes, prominent nucleoli, and increased mitotic activity. The cells are arranged in aggregates and diffuse sheets. Given the overlapping morphologic features, immunophenotypic analysis is considered essential to distinguish MS and BPDCN; however, these entities can share expression of many antigens, including CD4, CD14, CD33, CD43, CD68, and CD163. ${ }^{9-12}$ Three additional markers have been suggested as being helpful for distinguishing BPDCN from MSCLA/CD162, blood dendritic cell antigen (BDCA2)/ CD303, and interferon- $\alpha$-inducible protein myxovirus $\mathrm{A}(\mathrm{MxA})$.

In this study, our goal was to develop an optimized immunohistochemical panel to distinguish MS from BPDCN. We used a well-characterized cohort of cases assessed by clinical data and follow-up, as well as being assessed by numerous markers as part of the work up, to distinguish BPDCN from MS. We then studied a battery of immunohistochemical stains, including CD4, CD14, CD33, CD43, CD56, CD68, CD123, CD163, myeloperoxidase, lysozyme, terminal deoxynucleotidyl transferase (TdT), TCL1, MxA1, CLA/CD162, and CD303/BDCA2.

\section{Materials and methods}

Following Institutional Review Board approval, 23 cases of MS and 17 cases of BPDCN were identified as the study group. The selected ones were the cases reviewed independently by three hematopathologists (MES, NAS, and JLP), and only those where there was consensus about the diagnosis as per the World Health Organization (WHO) 2008 criteria, were included in the study. ${ }^{4}$ Clinical data related to age, sex, and tumor location were noted. The demographics and clinical site for each case included in the study are itemized in Table 1. The cases in our study involved predominantly skin, lymph node, and bone marrow, along with occasional cases involving uncommon sites such as soft tissue, testis, and meninges.

Table 1 Summary of patient and lesion characteristics

\begin{tabular}{lccr}
\hline Patient Characteristics & $M S$ & BPDCN & Total \\
\hline Age, mean & 38 years & 60 years & \\
Age, range & 4 months-78 years & $14-81$ years & \\
Gender (M/F) & $19 / 4$ & $13 / 4$ & $32 / 8$ \\
Sites & & & \\
$\quad$ Skin & 7 & 7 & 14 \\
Lymph node & 4 & 3 & 7 \\
$\quad$ Bone marrow & 0 & 3 & 4 \\
$\quad$ Other & 12 & 17 & 40 \\
Total & 23 & & \\
\hline
\end{tabular}

We reviewed hematoxylin and eosin stained sections and immunohistochemical studies performed at time of original diagnosis. The $23 \mathrm{MS}$ and 17 BPDCN were stained with a panel of 15 antibodies (Table 2). We could not test each case with the full set of stains due to sample limitations.

Immunohistochemical analysis was performed on $4-\mu$-thick sections of formalin-fixed (4-6 h, standard fixation time), paraffin-embedded tissues. Sections were air-dried at room temperature and then placed in a $60{ }^{\circ} \mathrm{C}$ oven for $30 \mathrm{~min}$ to melt the paraffin. Sections were deparaffinized in three changes of xylene ( $5 \mathrm{~min}$ each), rehydrated through graded alcohols for $1 \mathrm{~min}$ each $(100 \% \times 2,95 \% \times 2$, and $70 \%$ ), then placed in deionized $\mathrm{H}_{2} \mathrm{O}$. Next, heatinduced epitope retrieval was performed manually in citrate buffer, $\mathrm{pH}$ 6.0, in an electric pressure cooker for $4 \mathrm{~min}$ at $120^{\circ} \mathrm{C}$ (TCL1 and CD303) or in a microwave oven for $15 \mathrm{~min}$ at half power and then cooled in hot buffer for an additional 15 min (CLA/ CD162 and MxA). The sections were then placed in $\mathrm{dH}_{2} \mathrm{O}$ and loaded onto the automated immunostainer. All of the staining steps were performed at $37^{\circ} \mathrm{C}$ on the automated immunostainer (ULTRA) from Ventana Medical Systems, Tucson, AZ. CD4, CD14, CD33, CD43, CD56, CD68, CD123, myeloperoxidase (MPO), lysozyme (LYS), and TdT were all de-paraffized on the automated instrument with EZ Prep Solution (Ventana Medical Systems) then underwent antigen retrieval with CC1 (Cell Conditioning 1) or CC2 (Cell Conditioning 2) (see Table 2). Antibodies were applied to the slides. The sections were detected using the IView DAB detection kit (Ventana Medical Systems), a biotinylated goat antimouse/anti-rabbit secondary, Streptavidin-HRP system, utilizing DAB (3-3' diaminobenzidine) as

Table 2 Description of immunohistochemical antibodies

\begin{tabular}{lcll}
\hline Antibody & Clone & Dilution & Manufacturer \\
\hline CD4 & 1F6 & $1: 50$ & Leica/Novocastra \\
CD14 & 7 & $1: 50$ & Leica/Novocastra \\
CD33 & PWS44 & $1: 150$ & Leica/Novocastra \\
CD43 & L60 & $\begin{array}{l}\text { Pre- } \\
\text { dilute }\end{array}$ & Ventana Medical \\
& & $1: 50$ & Therms \\
CD56 & 123C3.D5 & $1: 1600$ & Dako \\
CD68 & KP1 & $1: 200$ & BD Bioscientific \\
CD123 & 7G3 & $1: 200$ & Leica/Novocastra \\
CD163 & 10D6 & $1: 1600$ & Dako \\
MPO & Polyclonal & $1: 3200$ & Dako \\
Lysozyme & Polyclonal & $1: 400$ & Supertechs \\
TdT & Rabbit anti-human, & & \\
TCL1 & no clone listed & $1: 1000$ & Upstate/Cell \\
& TCL1A isoform, amino & 100 & Signaling \\
CLA/ & acids 60-73 & & Solutions \\
CD162 & HECA-452 & $1: 100$ & Novus \\
CD303/ & Polyclonal & $1: 50$ & Sigma \\
BDCA2 & Polyclonal & $1: 200$ & Novus \\
MxA & & & \\
\hline
\end{tabular}


the chromogen. The sections were counterstained with hematoxylin (Ventana Medical Systems) for $8 \mathrm{~min}$. The sections were removed from the immunostainer and placed in a dH2O/DAWN mixture (1 $\mathrm{ml}$ of DAWN/ $500 \mathrm{ml}$ of dH2O). The sections were gently washed in the dH2O/DAWN mixture, to remove any coverslip oil applied by the automated instrument. The sections were (1) rinsed in dH2O until all of the dH2O/DAWN mixture was removed, (2) placed in iodine for $30 \mathrm{sec}$ to remove any precipitates from fixation, (3) dipped in sodium thiosulfate to clear the iodine, (4) dehydrated in graded alcohols $(70 \%, 95 \% \times 2$ and $100 \% \times 2)$, (5) cleared in xylene, and (6) cover slipped.

The results were scored independently by three hematopathologists (MES, NAS, and JLP) as follows: 1 for a positive ( $>30 \%$ positive staining), -1 for a negative stain, and 0 otherwise (not performed or equivocal). Associations between antibody results and reference diagnoses were evaluated by a $2 \times 2$ table for each marker (staining pattern vs reference diagnosis) using Fisher's exact test. Statistical significance was defined as 5\% (two-sided). Statistical calculations were performed using Stata version 12 (Stata Corp, College Station, TX, USA).

\section{Results}

Morphological analysis of the study cohort showed similar features for cases of MS and BPDCN with sheet-like infiltrates and aggregates of large atypical cells with a high nuclear-to-cytoplasmic ratio, irregular nuclear membranes, prominent nucleoli, and increased mitotic activity (Figures 1a and 2a). The results of the immunohistochemical studies are summarized in Table 3. Seven antibodies (CD4, CD56, CD123, lysozyme, myeloperoxidase, TCL1, and MxA) showed a statistically significant association (Fisher's exact test, $P<0.05$ ) with the reference diagnosis. Positive staining for CD4, CD56, CD123, TCL1, and MxA was associated with BPDCN. Positive staining for myeloperoxidase and lysozyme was associated with MS. The staining patterns for
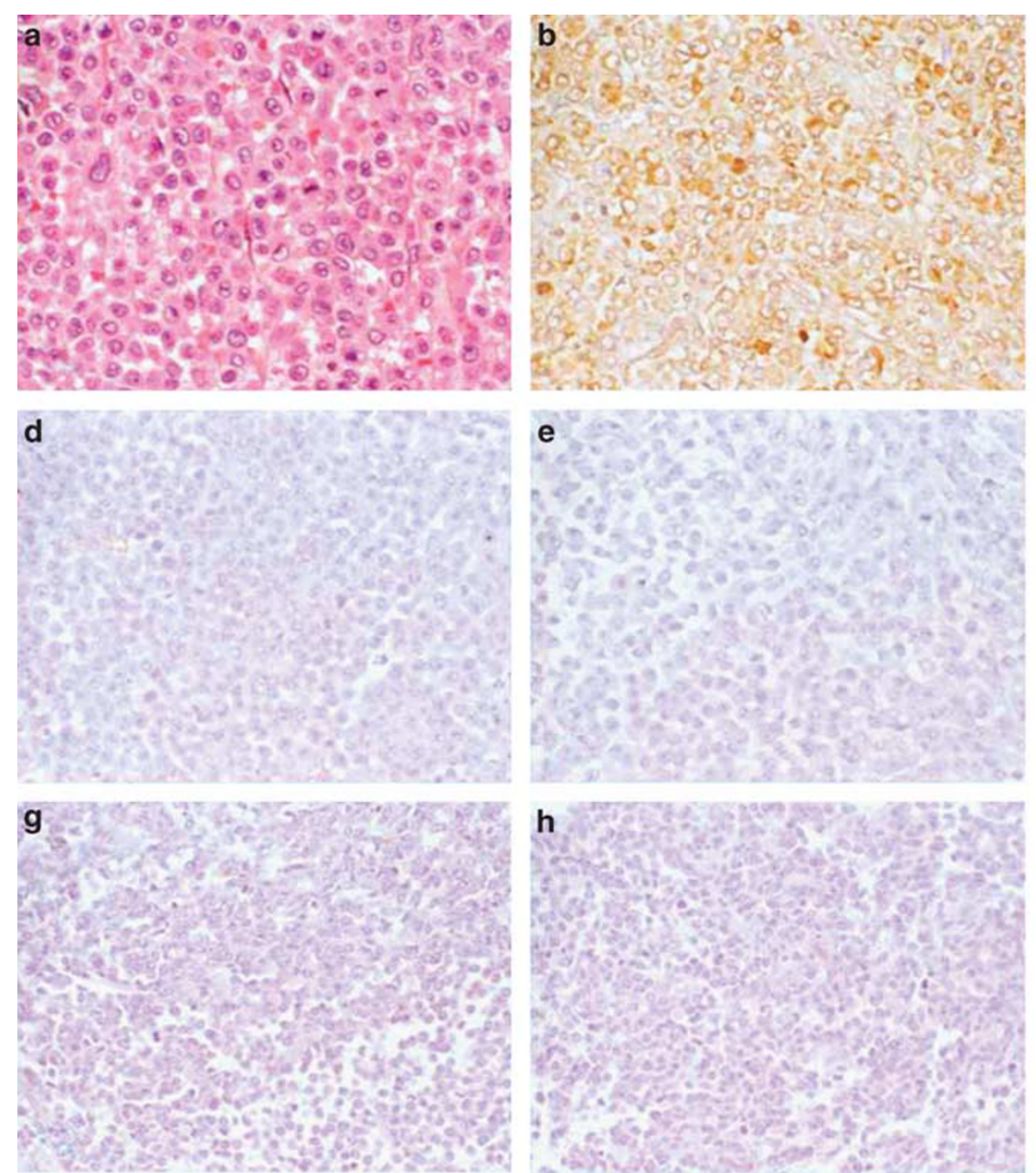

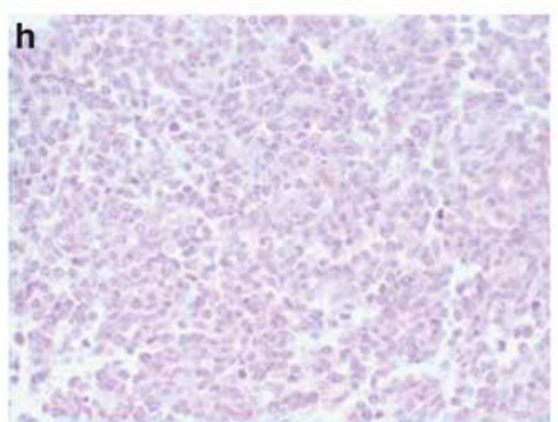

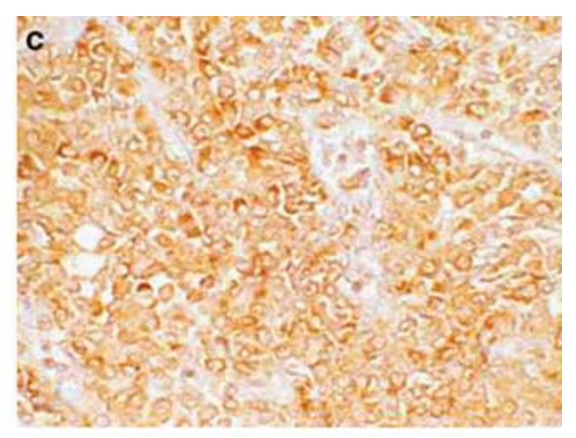

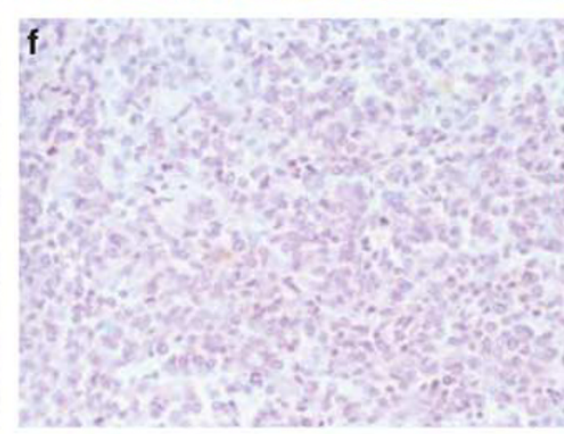

Figure 1 MS showing diffuse sheets of large atypical neoplastic cells with occasional prominent nucleoli and prominent mitotic activity (a), with positivity for lysozyme (b), and myeloperoxidase (c), and absence of staining for MxA, TCL1, TdT, CD56, and CD123 (d-h, respectively). 

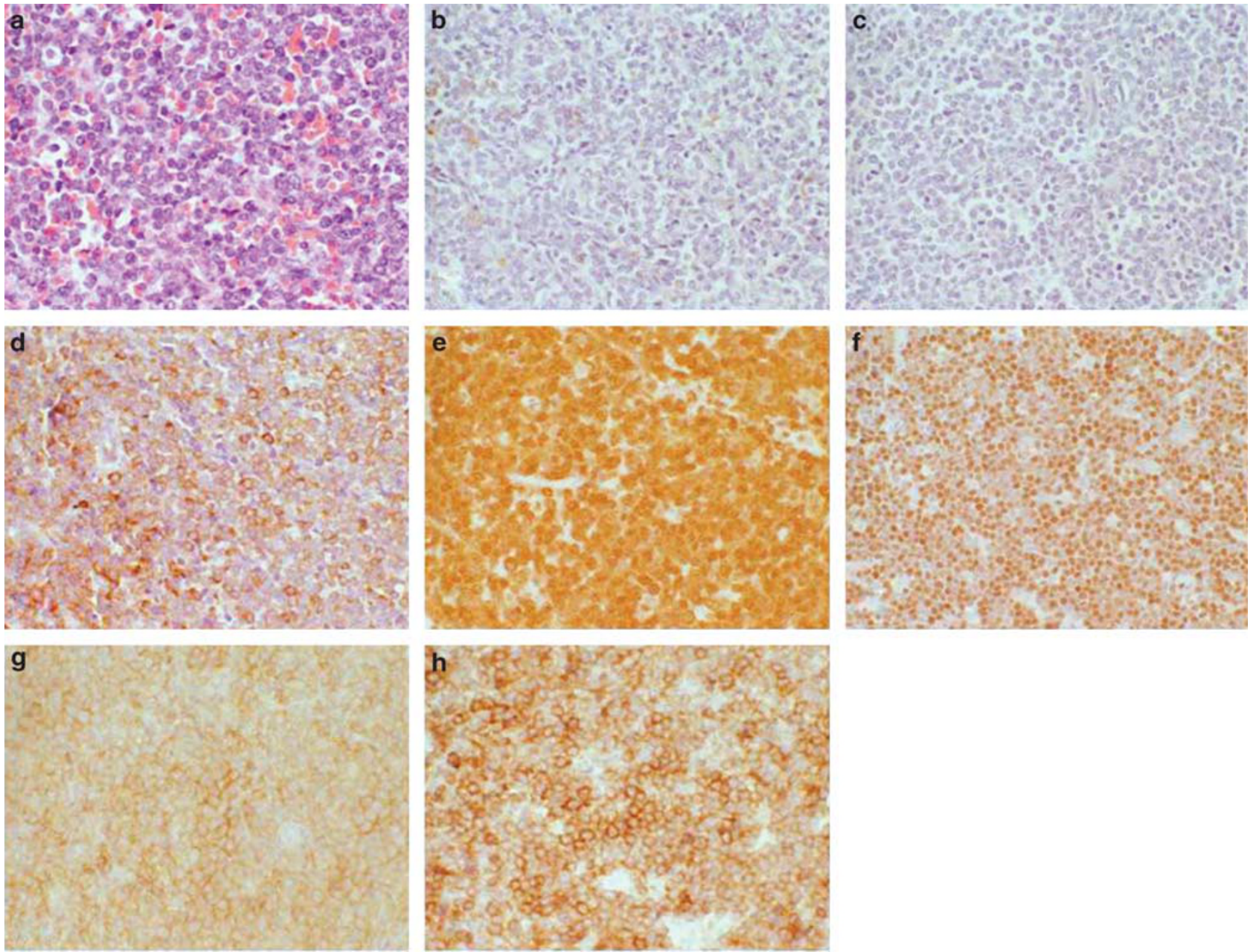

Figure 2 BPDCN histologically composed of a high-grade malignant neoplasm with diffuse sheets of large atypical cells with vesicular chromatin and inconspicuous nucleoli, and occasional atypical mitotic figures (a). The tumor shows no immunoreactivity for lysozyme (b) or myeloperoxidase (c); but exhibits intense staining for MxA (d), TCL1 (e), nuclear positivity for TdT (f), and membranous staining for CD56 (g) and CD123 (h).

CD4, CD56, CD123, TCL1, and MxA showed a significant positive correlation (Table 4). Myeloperoxidase and lysozyme showed a significant positive pairwise correlation but were negatively correlated with CD4, CD56, CD123, TCL1, and MxA. CLA/ CD162 and CD303 showed no statistically significant association with the established diagnoses. Positive staining for MxA was associated with BPDCN $(P=0.03)$. We found that positive staining for lysozyme or myeloperoxidase, or negative staining for CD4, CD56, CD123, MxA, or TCL1, were associated with MS (Figures 1b-h). BPDCN was associated with positive CD56, TdT, or TCL1, or negative lysozyme (Figures $2 \mathrm{~b}-\mathrm{h}$ ).

It was not possible to create a logistic regression model to predict BPDCN based on marker values due to colinearity, missing values, and zeros in the marginal distributions. Thus, an aggregate score was devised by assigning a weight of 1 for a positive result, -1 for a negative result, and 0 otherwise (not performed or equivocal) for each of the seven markers:
Seven-antibody $\quad$ score $=(C D 4+$ CD56 + CD123 + TCL1 + MxA)-(LYS + MPO)

As shown in Table 5, the seven-stain score was able to provide perfect discrimination between MS and BPDCN (area under the receiver operating characteristic curve $($ AUROC $)=1.00,95 \%$ confidence interval: 1.00-1.00).

A simpler scoring scheme based on two antibodies (CD56 and TCL1) also performed well (Table 6):

Two-antibody score: $=($ CD56 + TCL1 $)$

For the two-antibody score, a score of greater than or equal to zero was associated with BPDCN (AUROC $=0.997,95 \%$ confidence interval: 0.991.00).

\section{Discussion}

BPDCN and MS can be morphologically indistinguishable hematopoietic malignancies with a tropism for involving the skin. MS and BPDCN are 
Table 3 Staining results

\begin{tabular}{|c|c|c|c|c|c|c|c|c|}
\hline \multirow[b]{3}{*}{ Marker } & \multicolumn{4}{|c|}{ Stain results } & \multicolumn{2}{|c|}{ Likelihood ratio (for BPDCN) } & \multirow[b]{3}{*}{ AUROC } & \multirow[b]{3}{*}{$P$-value } \\
\hline & \multicolumn{2}{|c|}{ Negative } & \multicolumn{2}{|c|}{ Positive } & \multirow[b]{2}{*}{$L R+$} & \multirow[b]{2}{*}{$L R-$} & & \\
\hline & MS & $B P D C N$ & MS & $B P D C N$ & & & & \\
\hline CD4 & 14 & 0 & 4 & 17 & $4.1(1.8-10.7)$ & $0.04(0.002-0.6)$ & $0.89(0.79-0.99)$ & $<0.001$ \\
\hline CD14 & 7 & 9 & 1 & 1 & $0.8(0.1-6.6)$ & $1.0(0.7-1.5)$ & $0.49(0.33-0.64)$ & 1.00 \\
\hline CD33 & 1 & 1 & 17 & 9 & $0.9(0.7-1.2)$ & $1.7(0.2-14.6)$ & $0.48(0.37-0.59)$ & 1.00 \\
\hline CD43 & 0 & 0 & 12 & 3 & NC & NC & NC & NC \\
\hline CD56 & 19 & 0 & 1 & 17 & $13.6(2.9-63.7)$ & $0.03(0.002-0.5)$ & $0.98(0.93-1.00)$ & $<0.001$ \\
\hline CD68 & 1 & 1 & 11 & 4 & $0.8(0.5-1.4)$ & $2.2(0.3-16.8)$ & $0.44(0.23-0.65)$ & 0.52 \\
\hline CD123 & 10 & 0 & 3 & 16 & $3.9(1.6-9.7)$ & $0.04(0.003-0.6)$ & $0.88(0.76-1.00)$ & $<0.001$ \\
\hline CD163 & 7 & 9 & 3 & 0 & $0.2(0.009-2.7)$ & $1.4(0.9-2.1)$ & $0.35(0.20-0.50)$ & 0.21 \\
\hline LYS & 1 & 13 & 11 & 0 & $0.04(0.003-0.6)$ & $8.4(1.8-37.8)$ & $0.04(0-0.12)$ & $<0.001$ \\
\hline MPO & 6 & 13 & 11 & 0 & $0.07(0.004-0.9)$ & $2.67(1.4-5.0)$ & $0.2(0.06-0.3)$ & $<0.001$ \\
\hline $\mathrm{TdT}$ & 3 & 7 & 0 & 5 & $3.4(0.2-48.8)$ & $0.7(0.4-1.2)$ & $0.71(0.56-0.85)$ & 0.50 \\
\hline TCL1 & 11 & 1 & 0 & 9 & $20.7(1.4-316.0)$ & $0.14(0.03-0.6)$ & $0.95(0.85-1.00)$ & $<0.001$ \\
\hline CLA/CD162 & 10 & 5 & 0 & 2 & $6.9(0.4-125.0)$ & $0.72(0.4-1.2)$ & $0.64(0.46-0.82)$ & 0.15 \\
\hline CD303 & 10 & 5 & 0 & 2 & $6.9(0.4-125.0)$ & $0.72(0.4-1.2)$ & $0.64(0.46-0.82)$ & 0.15 \\
\hline MxA & 6 & 0 & 3 & 6 & $2.65(1.1-6.3)$ & $0.1(0.007-1.6)$ & $0.83(0.67-1.00)$ & 0.03 \\
\hline
\end{tabular}

Abbreviations: AUROC, area under the receiver operating characteristic curve; BPDCN, blastic plasmacytoid dendritic cell neoplasm; LR +, positive likelihood ratio; LR -, negative likelihood ratio; LYS, lysozyme; MPO, myeloperoxidase; MS, myeloid sarcoma.

Each entry under 'stain results' indicates the number of cases for each stain and the corresponding reference diagnosis (MS vs BPDCN). $P$-values correspond to Fisher's exact test for each stain vs reference diagnosis.

Table 4 Correlations between marker positivity

Stain 2

\begin{tabular}{|c|c|c|c|c|c|c|c|}
\hline Stain 1 & $C D 4$ & $C D 56$ & CD123 & $M P O$ & $L Y S$ & TCL1 & $M \times A$ \\
\hline CD4 & 1.00 & & & & & & \\
\hline CD56 & $0.77(0.00)$ & 1.00 & & & & & \\
\hline CD123 & $0.69(0.00)$ & $0.84(0.00)$ & 1.00 & & & & \\
\hline MPO & $-0.33(0.10)$ & $-0.75(0.00)$ & $-0.73(0.00)$ & 1.00 & & & \\
\hline LYS & $-0.70(0.00)$ & $-0.83(0.00)$ & $-0.56(0.01)$ & $0.57(0.01)$ & 1.00 & & \\
\hline TCL1 & $0.71(0.00)$ & $0.81(0.00)$ & $0.61(0.02)$ & $0.58(0.02)$ & $\begin{array}{c}-0.89 \\
0.00\end{array}$ & 1.00 & \\
\hline MxA & $0.66(0.02)$ & $0.85(0.00)$ & $0.54(0.69)$ & $-0.41(0.20)$ & $-0.73(0.00)$ & $0.67(0.01)$ & 1.00 \\
\hline
\end{tabular}

Abbreviations: LYS, lysozyme; MPO, myeloperoxidase.

Each entry indicates Pearson's correlation coefficient between the scores $(1=$ positive, $0=$ negative $)$ for each marker and the associated $P$-value. For example, the correlation coefficient between LYS and CD123 is $-0.56(P=0.01)$.

biologically different entities with well-characterized cell(s) of origin and are broadly classified under the heading of 'acute myeloid leukemia and related precursor neoplasms' in the WHO 2008 classification. ${ }^{4}$ These are relatively rare neoplasms, and their diagnosis can be particularly challenging on morphological and clinical grounds. Immunohistochemical panels have been proposed in the literature to distinguish the two entities; however, an extended immunohistochemical panel including relatively recently described immunomarkers such as CLA/CD162, CD303/BDC2 and MxA has not been studied. Recent literature suggests that differentiating MS from BPDCN has prognostic implications and is important for therapeutic decisions. ${ }^{13,14}$ MSs are classified cytogenetically and immunophe- notypically, in the same manner as if they involved the bone marrow and are treated accordingly. The diagnosis of BPDCN mandates aggressive chemotherapy, with or without bone marrow or stem cell transplant. ${ }^{13-15}$

In this study using immunohistochemical analysis, we found that seven antibodies are useful for distinguishing between MS from BPDCN: CD4, CD56, CD123, TCL1, lysozyme, MxA, and myeloperoxidase (Table 3). Positive staining for lysozyme or myeloperoxidase, or negative results for CD4, CD56, CD123, MxA, or TCL1, was associated with MS (Figures 1b-h). Cases of BPDCN were associated with any of the following: positive CD56, TdT, or TCL1, or negative staining for lysozyme (Figures $2 \mathrm{~b}-\mathrm{h}$ ). A simple scoring system based on these results that 
Table 5 Differentiation of BPDCN from MS by immunohistochemical score using seven stains

\begin{tabular}{cccc}
\hline & \multicolumn{2}{c}{ Diagnosis } \\
\cline { 2 - 3 } Score & MS & TPDCN & \\
\hline-6 & 1 & 0 & 1 \\
-5 & 1 & 0 & 1 \\
-4 & 6 & 0 & 6 \\
-3 & 7 & 0 & 7 \\
-2 & 3 & 0 & 3 \\
-1 & 3 & 0 & 3 \\
0 & 1 & 0 & 1 \\
1 & 1 & 0 & 1 \\
2 & 0 & 1 & 1 \\
3 & 0 & 2 & 2 \\
4 & 0 & 1 & 6 \\
5 & 0 & 6 & 1 \\
6 & 0 & 1 & 6 \\
7 & 0 & 6 & 40 \\
Total & 23 & 17 & \\
\hline
\end{tabular}

Abbreviations: BPDCN, blastic plasmacytoid dendritic cell neoplasm; LYS, lysozyme; MPO, myeloperoxidase; MS, myeloid sarcoma.

Score $=($ CD4 + CD56 + CD123 + TCL1 + MxA $)-($ LYS + MPO $)$ where the individual scores were 1 if positive, -1 if negative and 0 otherwise (not performed or equivocable). For example, CD4 = pos, $\mathrm{CD} 56=$ neg, $\mathrm{CD} 123=$ pos, $\mathrm{TCL} 1=$ not measured MxA $=$ neg, $\mathrm{LYS}=$ neg and MPO $=$ neg would give a score of $1-1+1+0-1-(-1-1)$ $=2$. MS is associated with lower scores and BPDCN is associated with higher scores. For this data set, a cutoff score of greater than one perfectly predicts BPDCN.

Table 6 Differentiation of BPDCN from MS by immunohistochemical score using two stains

\begin{tabular}{lrrr}
\hline & \multicolumn{2}{c}{ Diagnosis } \\
\cline { 2 - 3 } Score & $M S$ & BPDCN & Total \\
\hline-2 & 8 & 0 & 8 \\
-1 & 13 & 0 & 13 \\
0 & 2 & 7 & 3 \\
1 & 0 & 9 & 7 \\
2 & 0 & 17 & 9 \\
Total & 23 & &
\end{tabular}

Abbreviations: BPDCN, blastic plasmacytoid dendritic cell neoplasm; LYS, lysozyme; MPO, myeloperoxidase; MS, myeloid sarcoma. Score $=$ Score $=($ CD56 + TCL 1 where the individual scores were 1 if positive, -1 if negative, and 0 otherwise (not performed or equivocal). For example, CD56 $=$ neg, TCL1 $=$ neg would give a score of -2 . MS is associated with lower scores and BPDCN is associated with higher scores. For this dataset, a cutoff score of greater than one predicts BPDCN.

incorporates CD4, CD56, CD123, TCL1, MxA, lysozyme, and myeloperoxidase was able to perfectly discriminate between MS and BPDCN (Table 5). Using only CD56 and TCL1 was able to provide nearly equivalent discrimination between BPDCN and MS and may be more cost effective (Table 6). CD68 and lysozyme have been described as the most consistently expressed and sensitive immunohistochemical stains in MS. ${ }^{16}$ Myeloperoxidase is considered to be a specific marker; however, it is positive in only 58\% cases. CD4 and CD56 are only rarely positive in MS. ${ }^{16}$ Most BPDCN cases described in the literature have been CD4- and CD56-positive; however, rare cases negative for both of these markers have been reported. ${ }^{17-19}$ Expression of CD123 by BPDCN cases supports their origin from immature plasmacytoid dendritic cells, and most cases of BPDCN have shown to exhibit intense staining for CD123. In MS, CD123 expression has been reported in a subset of cases, ranging from 9 to $45 \% .{ }^{9}$ Herling et $a .^{20}$ have shown the near universal presence of TCL-1 in BPDCN, however, Petrella et al. ${ }^{7}$ have described TCL1 expression in 17\% of MS cases.

In our study, we found that myeloperoxidase and lysozyme were negative in all the cases of BPDCN. In MS, myeloperoxidase was positive in 12/18 (67\%), whereas lysozyme was positive in 11/12 (91\%) cases. All BPDCN cases were positive for CD4 and CD56, in contrast, CD4 and CD56 were positive in 4/18 $(22 \%)$ and $1 / 20(5 \%)$ MS cases, respectively. CD123 was positive in all cases of BPDCN $(n=17)$ and showed immunoreactivity in only $3 / 16(19 \%)$ MS cases. TCL1 was observed in all 11 BPDCN cases and not in any of 12 MS cases. These results are similar to a recently published study by Cronin et al, ${ }^{21}$ where CD4, CD56, CD123, and TCl-1 are described as the most useful markers to distinguish between myeloperoxidase-negative MS and BPDCN. ${ }^{21}$

In addition to the established antibodies discussed above, we also evaluated three relatively recently described immunohistochemical antibodies that have been reported to be expressed in cases of BPDCN: CLA/CD162, BDCA2/CD303, and MxA. Of these, only MxA showed a statistically significant association with the reference diagnoses $(P=0.03)$. The results for these studies, however, are limited by small sample size $(n<17)$.

CLA/CD162 is a cell surface glycoprotein that is thought to have a role in lymphocyte homing to skin. ${ }^{22}$ Petrella et $a l^{7}$ studied CLA/CD162 and found it to be positive in $90 \%$ of BPDCN and $78 \%$ of MS cases, suggesting the lack of specificity of this marker in distinguishing MS and BPDCN. In contrast, CLA/CD162 was positive in 3/9 (33\%) BPDCN cases and in 0/11 $(0 \%)$ cases of MS in our study. BDCA2/CD303 is a cell surface protein which is expressed preferentially on human plasmacytoid dendritic cells. ${ }^{23}$ The expression of this antigen on BPDCN has been previously described in the research literature; ${ }^{23,24}$ however, its utility has not been studied clinically. In this study, we found BDCA2/CD303 in 0/11 (0\%) MS cases and in 2/10 $(20 \%)$ of BPDCN cases.

MxA is an interferon- $\alpha$-inducible protein, the positivity for which suggests interferon- $\alpha$ production, characteristic of plasmacytoid dendritic cells. ${ }^{24}$ Immunoreactivity of blastic plasmacytoid dendritic cells for MxA antibody has been described by Pilichowska et al. ${ }^{24}$ However, this antibody also has not been formally assessed in a clinical setting to distinguish MS and BPDCN. We found that 3/9 (33\%) 
MS cases were positive for MxA, in contrast with 8/8 (100\%) BPDCN cases. As far as the authors are aware, this is the first published study where the clinical utility of CLA/CD162, CD303/BDC2, and MxA have been studied in MS and BPDCN.

In summary, distinguishing BPDCN from MS in extranodal sites such as skin is often difficult because of extensive overlap of clinical and morphologic features. Immunophenotypic studies are needed and at extranodal sites immunohistochemical analysis is often the best choice as fresh tissue is not available. The results of this study show that positive staining for myeloperoxidase or lysozyme and negative results for CD56, CD123, TCL1, or MxA predict the diagnosis of MS reliably (100\%). Positive staining for CD56, TdT, or TCL1 and negative results for lysozyme predict the diagnosis of BPDCN (90\%). Other markers, including CLA/CD162 and CD303/ BDC2, may also have value but as they reacted with only small subsets of cases, these markers had low predictive value in this study.

\section{Disclosure/conflict of interest}

The authors declare no conflict of interest.

\section{References}

1 Adachi M, Maeda K, Takekawa M, et al. High expression of CD56 (N-CAM) in a patient with cutaneous CD4-positive lymphoma. Am J Hematol 1994;47:278-282.

2 Chaperot L, Bendriss N, Manches O, et al. Identification of a leukemic counterpart of the plasmacytoid dendritic cells. Blood 2001;97:3210-3217.

3 Petrella T, Comeau MR, Maynadie M, et al. 'Agranular $\mathrm{CD} 4+\mathrm{CD} 56+$ hematodermic neoplasm' (blastic NK-cell lymphoma) originates from a population of CD56 + precursor cells related to plasmacytoid monocytes. Am J Surg Pathol 2002;26:852-862.

4 Stein H, Warnke RA, Chan WC. Diffuse large B-cell lymphoma, not otherwise specified, In: Swerdlow SH, Campo E, Harris NL et al. (eds). WHO Classification of Tumors of Haematopoietic and Lymphoid Tissues. 4th edn. IARC Press: Lyon, France; 2008, pp 233-239.

5 Urosevic M, Conrad C, Kamarashev J, et al. CD4+ CD56 + hematodermic neoplasms bear a plasmacytoid dendritic cell phenotype. Hum Pathol 2005;36: 1020-1024.

6 Petrella T, Bagot M, Willemze R, et al. Blastic NK-cell lymphomas (agranular CD4 + CD56 + hematodermic neoplasms): a review. Am J Clin Pathol 2005;123: 662-675.

7 Petrella T, Meijer CJ, Dalac S, et al. TCL1 and CLA expression in agranular CD4/CD56 hematodermic neoplasms (blastic NK-cell lymphomas) and leukemia cutis. Am J Clin Pathol 2004;122:307-313.

8 Menasce LP, Banerjee SS, Beckett E, et al. Extramedullary myeloid tumour (granulocytic sarcoma) is often misdiagnosed: a study of 26 cases. Histopathology 1999;34:391-398.
9 Benet C, Gomez A, Aguilar C, et al. Histologic and immunohistologic characterization of skin localization of myeloid disorders: a study of 173 cases. Am J Clin Pathol 2011;135:278-290.

10 Roth MJ, Medeiros LJ, Elenitoba-Johnson K, et al. Extramedullary myeloid cell tumors. An immunohistochemical study of 29 cases using routinely fixed and processed paraffin-embedded tissue sections. Arch of Pathol Lab Med 1995;119:790-798.

11 Ratnam KV, Su WP, Ziesmer SC, et al. Value of immunohistochemistry in the diagnosis of leukemia cutis: study of 54 cases using paraffin-section markers. J Cutan Pathol 1992;19:193-200.

12 Kaddu S, Zenahlik P, Beham-Schmid C, et al. Specific cutaneous infiltrates in patients with myelogenous leukemia: a clinicopathologic study of 26 patients with assessment of diagnostic criteria. J Am Acad Dermatol 1999;40:966-978.

13 Tsagarakis NJ, Kentrou NA, Papadimitriou KA, et al. Acute lymphoplasmacytoid dendritic cell (DC2) leukemia: results from the Hellenic Dendritic Cell Leukemia Study Group. Leukemia Res 2010;34:438-446.

14 Dalle S, Beylot-Barry M, Bagot M, et al. Blastic plasmacytoid dendritic cell neoplasm: is transplantation the treatment of choice? Br J Dermatol 2010;162:74-79.

15 Dietrich S, Andrulis M, Hegenbart U, et al. Blastic plasmacytoid dendritic cell neoplasia (BPDC) in elderly patients: results of a treatment algorithm employing allogeneic stem cell transplantation with moderately reduced conditioning intensity. Biol Blood Marrow Transplant 2011;17:1250-1254.

16 Cibull TL, Thomas AB, O'Malley DP, et al. Myeloid leukemia cutis: a histologic and immunohistochemical review. J Cutan Pathol 2008;35:180-185.

17 Ascani S, Massone C, Ferrara G, et al. CD4-negative variant of $\mathrm{CD} 4+/ \mathrm{CD} 56+$ hematodermic neoplasm: description of three cases. J Cutan Pathol 2008;35:911-915.

18 Argyrakos T, Rontogianni D, Karmiris T, et al. Blastic natural killer (NK)-cell lymphoma: report of an unusual CD4 negative case and review of the CD4 negative neoplasms with blastic features in the literature. Leukemia Lymphoma 2004;45:2127-2133.

19 Cota C, Vale E, Viana I, et al. Cutaneous manifestations of blastic plasmacytoid dendritic cell neoplasm-morphologic and phenotypic variability in a series of 33 patients. Am J Surg Pathol 2010;34:75-87.

20 Herling M, Teitell MA, Shen RR, et al. TCL1 expression in plasmacytoid dendritic cells (DC2s) and the related CD4 + CD56 + blastic tumors of skin. Blood 2003;101:5007-5009.

21 Cronin DM, George TI, Reichard KK, et al. Immunophenotypic analysis of myeloperoxidase-negative leukemia cutis and blastic plasmacytoid dendritic cell neoplasm. Am J Clin Pathol 2012;137:367-376.

22 Tsuchiyama J, Yoshino T, Toba K, et al. Induction and characterization of cutaneous lymphocyte antigen on natural killer cells. Br J Haematol 2002;118:654-662.

23 Jaye DL, Geigerman CM, Herling M, et al. Expression of the plasmacytoid dendritic cell marker BDCA-2 supports a spectrum of maturation among CD4 + CD56 + hematodermic neoplasms. Mod Pathol 2006;19:1555-1562.

24 Pilichowska ME, Fleming MD, Pinkus JL, et al. CD4 + / $\mathrm{CD} 56+$ hematodermic neoplasm ("blastic natural killer cell lymphoma"): neoplastic cells express the immature dendritic cell marker BDCA-2 and produce interferon. Am J Clin Pathol 2007;128:445-453. 